(2)

\title{
Associação entre consumo de álcool, quedas e internação em idosos
}

\author{
Gleiverson S. Sequeto, ${ }^{1}$ Natalia A. Santos, ${ }^{1}$ Lilian Atalaia-Silva, ${ }^{2}$ Cláudia Helena C. Mármora ${ }^{1 *}$
}

\section{Resumo}

Introdução: O envelhecimento associado a perdas cognitivas, como velocidade de processamento e tempo de reação, e ao alto consumo de álcool aumenta o risco de quedas tendo como desfecho a internação. Objetivo: Analisar a associação entre o consumo alcoólico de risco e quedas em idosos, relacionando a incidência de quedas a períodos de internações anteriores verificando diferenças no consumo alcoólico de risco entre homens e mulheres. Materiais e métodos: Estudo do tipo transversal, descritivo, utilizando dados do projeto FIBRA-JF com idosos que declararam consumo de álcool. Foram analisados dois subgrupos (M e F) relacionando o consumo de risco com quedas e internação por pelo menos uma noite nos últimos doze meses. Resultados: A amostra composta por 128 idosos que relataram consumir álcool foi de 73 mulheres e 55 homens. Os resultados demonstraram que não houve diferença significativa para o consumo de risco de bebidas alcoólicas tanto nos homens ( $\mathrm{n}=16,51 \%)$ quanto nas mulheres $(n=37,16 \%)$ com $(p \leq 0,05)$. Não houve correlação entre consumo de risco e quedas nos homens ( $p=69,41 \%$ ), sendo observada diferença estatisticamente significativa nas mulheres, com $\mathrm{p}=99,55 \%$ ). Os idosos que consumiram de 2 a 3 doses por dia sofreram 3 quedas nos últimos 12 meses e aqueles que consumiram de 4 a 5 doses, sofreram 2 quedas nos últimos 12 meses. O consumo de risco de álcool em homens e mulheres associado à internação por pelo menos uma noite não apresentou relevância estatisticamente significativa. Com relação ao número de doses consumidas entre homens e mulheres, não se atingiu o nível de significância estabelecido inicialmente ( $\mathrm{p}=0,07$, para $\mathrm{p} \leq 0,05$ ), sendo que os homens ingerem maior quantidade de bebidas alcoólicas em relação às mulheres. Conclusões: O consumo de risco de bebidas alcoólicas em idosos pode estar relacionado com maior ocorrência de quedas nesta população, principalmente no gênero feminino devido a diversos fatores biológicos. Sendo assim, as mulheres possuem maior predisposição a sofrer quedas.

Descritores: Acidentes por quedas; Alcoolismo; Envelhecimento.

\section{Abstract \\ The association of alcohol consumption, falls, and hospitalization in elderly}

Introduction: Aging, associated with cognitive losses such as processing speed and reaction time, and with high alcohol consumption, increases the risk of falls resulting
1. Faculdade de Fisioterapia. Universidade Federal de Juiz de Fora. Juiz de Fora, MG, Brasil.

2. Departamento de Fisioterapia. Centro Universitário Estácio Juiz de Fora - UNESA. Juiz de Fora, MG, Brasil.

*Endereço para correspondência: Faculdade de Fisioterapia, UFJF.

Av. Eugênio do Nascimento s/n은

Juiz de Fora, MG, Brasil. CEP: 36038-330.

E-mail: claudia.marmora@ufj.edu.br

Revista HUPE, Rio de Janeiro, 2018;17(2):43-50

doi: $10.12957 /$ rhupe.2018.40810

Recebido em 07/05/2018. Aprovado em 06/11/2018.

in hospitalization. Objective: To analyze the association between risk-level alcohol consumption and falls in the elderly, relating the incidence of falls to periods of previous hospitalizations, verifying differences in the risk-level consumption of alcohol between men and women. Materials and methods: A cross-sectional descriptive study, with data from the FIBRA-JF project, with elderly individuals who reported alcohol consumption. Two subgroups ( $\mathrm{M}$ and $\mathrm{F}$ ) were analyzed, relating risk-level alcohol consumption with falls and hospitalization for at least one night in the last twelve months. Results: The sample composed of 128 elderly individuals who reported consuming alcohol had 73 women and 55 men. The results showed there was no significant difference in the consumption of alcoholic beverages in men $(n=16.51 \%)$ and in women $(n=37.16 \%)$ with $(\mathrm{p} \leq 0.05)$. There was no correlation between risk consumption and falls in men ( $\mathrm{p}=69.41 \%)$, with a statistically significant difference in women, with $\mathrm{p}=99.55 \%$ ). The elderly who consumed 2 to 3 doses per day suffered 3 falls in the last 12 months, and those who consumed 4 to 5 doses suffered 2 falls in the last 12 months. Risk-level alcohol consumption in men and women associated with hospitalization for at least one night did not present statistically significant significance. Regarding the number of doses consumed between men and women, a significance level of $\mathrm{p}=0.07(\mathrm{p} \leq 0.05)$ was observed, with men ingesting more alcoholic beverages than women. Conclusions: Risk-level alcohol consumption in the elderly may be related to a higher occurrence of falls in this population, mainly in the female gender due to several biological factors. Therefore, women are more likely to fall.

Keywords: Accidental falls; Alcoholism; Aging. 


\section{Artigo original}

\section{Resumen}

\section{Asociación entre consumo de alcohol, quedas y internación en idosos}

Introducción: el envejecimiento asociado a pérdidas cognitivas como la velocidad de procesamiento, el tiempo de reacción y el alto consumo de alcohol aumenta el riesgo de caídas que tienen como resultado la internación. Objetivo: analizar la asociación entre el consumo de riesgo de alcohol y caídas en ancianos, relacionando la incidencia de caídas a períodos de internaciones anteriores verificando diferencias en el consumo de riesgo de alcohol entre hombres y mujeres. Materiales y métodos: estudio del tipo transversal, descriptivo con los datos del proyecto FIBRA-JF con ancianos que declararon consumo de alcohol. Se analizaron dos subgrupos ( $\mathrm{M}$ y F) relacionando el consumo de riesgo con caídas e internación por al menos una noche en los últimos doce meses. Resultados: La muestra compuesta por 128 ancianos que relataron consumir alcohol fue de 73 mujeres y 55 hombres. Los resultados demostraron que no hubo diferencia significativa para el consumo de riesgo de bebidas alcohólicas tanto en los hombres ( $\mathrm{n}=16,51 \%)$ y en las mujeres $(\mathrm{n}=37,16 \%)$ $\operatorname{con}(\mathrm{p} \leq 0,05)$. No hubo correlación entre consumo de riesgo y caídas en los hombres $(\mathrm{p}=69,41 \%)$, siendo observada diferencia estadísticamente significativa en las mujeres, con $\mathrm{p}=99,55 \%$ ). Los ancianos que consumieron de 2 a 3 dosis al día, sufrieron 3 caídas en los últimos 12 meses y aquellos que consumieron de 4 a 5 dosis, sufrieron 2 caídas en los últimos 12 meses. El consumo de riesgo de alcohol en hombres y mujeres asociado a la internación por al menos una noche no tuvo relevancia estadísticamente significativa. En cuanto al número de dosis consumidas entre hombres y mujeres se observó una significación de $\mathrm{p}=0,07$ ( $\mathrm{p} \leq 0,05$ ), siendo que los hombres ingieren mayor cantidad de bebidas alcohólicas en relación a las mujeres. Conclusiones: el consumo de riesgo de bebidas alcohólicas en ancianos puede estar relacionado con mayor ocurrencia de caídas en esta población, principalmente en el género femenino debido a diversos factores biológicos. Siendo así las mujeres poseen mayor predisposición en sufrir caídas.

Palabras clave: Accidentes por caídas; Alcoholismo; Envejecimiento.

\section{Introdução}

O consumo de álcool é um dos hábitos sociais mais antigos e disseminados entre as populações, devido à sua relação com ritos religiosos. Dentre seus principais e variados efeitos podem ser referidos o de calmante, afrodisíaco, estimulante do apetite, desinibidor, entre outros. ${ }^{1}$

Segundo a Organização Mundial de Saúde(OMS) o consumo de risco de álcool está relacionado a maiores agravos e danos à saúde em geral. Os dados do relatório divulgado em 2017 indicam mudanças positivas entre os anos de 2007 e 2016, mostrando redução do consumo total em 3,5 L de álcool puro por pessoa, uma queda no número de novos casos diagnosticados como psicose alcoólica (de 52,3 para 20,5 por 100.000 habitantes) e por último uma queda da taxa de mortalidade de homens. Para o cálculo desse consumo, primeiro multiplica-se o número de doses ingeridas em um dia típico de consumo pelo número de dias em que se consumiram bebidas alcoólicas. Depois, divide-se o produto desta multiplicação por 30 (caso tenha sido relatado consumo mensal) ou 7 (para consumo semanal). Desta forma, chega-se a um consumo diário médio. Classifica-se como de risco o consumo diário médio acima das recomendações da OMS, ou seja, mais de duas doses padronizadas de bebidas para homens e mais de uma dose padronizada para mulheres, por dia. ${ }^{2}$

De acordo com o National Institute of Alcohol Abuse and Alcoholism (NIAAA, 2008), a definição de consumo moderado pode variar de acordo com o indivíduo e o contexto. O NIAAA aponta que dificuldades relacionadas à definição de uso moderado de álcool é até certo ponto o resultado das diferenças individuais, ou seja, a quantidade de álcool que uma pessoa pode consumir sem se intoxicar varia de acordo com a experiência, tolerância, metabolismo, vulnerabilidade genética, estilo de vida e tempo ao longo do qual o álcool é consumido. ${ }^{3}$

Os fatores de risco comportamentais ou comportamentos de risco incluem ações humanas, emoções ou escolhas diárias e são potencialmente modificáveis. O consumo de risco de álcool é um exemplo, sendo mais comum em homens do que em mulheres e, apesar dos homens indicarem uma predominância quanto à dependência do álcool, a população feminina tende a sofrer de forma mais intensa os danos cerebrais e físicos decorrentes da dependência alcoólica. ${ }^{4}$ Do ponto de vista biológico, as mulheres são metabolicamente menos tolerantes ao álcool do que os homens. ${ }^{5}$

Atualmente, o consumo de álcool tem aumentado entre mulheres que apresentam papéis sociais similares aos do homem. ${ }^{6,7}$ Mulheres mais jovens apresentam 
mais expectativas positivas em relação ao consumo quando comparadas às mais velhas, possivelmente porque o papel feminino está em mudança, com a mulher ocupando, cada vez mais, posições tradicionalmente masculinas. ${ }^{8,9}$ Fatores culturais e sociais reprimem mais o beber incompulsivo nas mulheres do que nos homens. Existe não só uma pressão social menor para que ela inicie a ingestão do álcool, mas também uma pressão maior para que ela interrompa o seu uso quando excessivo. ${ }^{10}$

O consumo de álcool é prevalente entre idosos mais longevos, e encontra-se associado com maior número de comorbidades, ao esquecimento em tomar os medicamentos prescritos e à grande preocupação de familiares e cuidadores com a ocorrência de quedas e fraturas. ${ }^{11}$

É conhecido que o envelhecimento acarreta perda cognitiva em determinadas funções cerebrais como a velocidade de processamento e o tempo de reação e, quando associado ao alto consumo de álcool, parece interferir mais rápido e negativamente no desempenho cognitivo, repercutindo na atenção visoespacial. Fatores que possam prejudicar esta função, como o álcool, aumentam a chance de quedas em idosos. ${ }^{12}$ Sendo este um evento fortemente vinculado ao envelhecimento, ele contribui diretamente para a morbidade e mortalidade nessa população. ${ }^{13,14}$

À medida que o corpo envelhece, alterações fisiológicas aumentam os efeitos nocivos do álcool e outras drogas. O aumento da incidência de fraturas em indivíduos que consomem álcool pode ser explicado pela ocorrência de quedas durante o período de intoxicação, ou por uma diminuição da densidade óssea em indivíduos alcoolistas. ${ }^{12}$

Embora o consumo total de álcool seja geralmente menor na população idosa, as alterações fisiológicas inerentes ao processo de envelhecimento aumentam seu efeito nocivo no organismo. ${ }^{17}$ Neste contexto, a relação entre o consumo de álcool e o risco de quedas representa uma ameaça significativa e potencialmente grave de acordo com a severidade da lesão provocada pela queda e a capacidade de recuperação. Muitas vezes são necessárias cirurgias ortopédicas com a colocação de próteses com longos períodos de imobilização e hospitalização. ${ }^{18}$

Nesta perspectiva, as quedas em idosos são atualmente uma importante questão de saúde pública devido à sua alta incidência e inevitáveis consequências, por muitas vezes irreparáveis não só para os idosos, mas também para a família. Além disso, elas representam um desafio para os serviços de saúde na qualificação de seus profissionais para o atendimento desta nova demanda. ${ }^{19,20}$

O consumo de álcool é a terceira causa de doença e morte em idosos internados e, apesar da variedade de consumo em quantidade e frequência, pode ser associado ao declínio na saúde física e/ou mental, causando isolamento social e comprometimento cognitivo. ${ }^{21,22}$

Este estudo objetiva verificar a existência de associação entre o uso abusivo de álcool e quedas em idosos com características de fragilidade. Ao se identificar fatores de risco que possam causar agravos à saúde da população idosa, como consumo de álcool, internações e quedas, torna-se possível buscar medidas de prevenção e intervenção que minimizem tais eventos, proporcionando uma melhor qualidade de vida para esta população. ${ }^{23-25}$

\section{Método}

Esta pesquisa foi desenvolvida a partir do banco de dados do projeto multicêntrico rede FIBRA Brasil de delineamento transversal e analítico realizado pelo polo UERJ com a população idosa do município de Juiz de Fora. ${ }^{26}$ Foram respeitados todos os padrões éticos aprovados pela Comissão Nacional de Ética em Pesquisa do Ministério da Saúde (CONEPE/MS - 313/2008) sob o processo número 555087/2006-9 com o apoio do Conselho Nacional de Desenvolvimento Científico e Tecnológico (CNPq). A coleta de dados ocorreu nos anos 2008-2009, nas residências dos idosos, e foi realizada por pesquisadores da Universidade Federal de Juiz de Fora devidamente treinados. ${ }^{26}$

Composição da amostra e seleção das variáveis do banco de dados para análise

A partir do banco FIBRA-JF foram incluídos os idosos que declararam consumo de álcool, sendo criados dois sub-bancos (masculino e feminino).

Em cada sub-banco foram feitas duas análises estatísticas. A primeira relacionando o fato de ser consumidor de risco e ter sofrido quedas nos últimos doze meses. E a segunda, ser consumidor de bebidas alcoólicas e ter sido internado por pelo menos uma noite.

\section{Instrumentos}

Para o presente estudo foi selecionada como variável independente o consumo de risco de álcool e como variáveis dependentes, quedas e internação para a caracterização da amostra, contendo itens como 


\section{Artigo original}

idade, gênero, cor da pele (auto relatada), estado civil, escolaridade e nível socioeconômico.

Além disso, foram utilizados os itens 90 "Quantas doses de bebidas alcoólicas o(a) sr(a) consome num dia normal?" e 91 "Com que frequência o sr (a) consome cinco ou mais doses em uma única ocasião?", referentes ao Alcohol Use Disorder Identification Test (AUDIT), instrumento utilizado para o rastreamento do uso problemático de álcool em serviços de saúde desenvolvido pela OMS..$^{27,28} \mathrm{O}$ instrumento original é composto por dez questões e as respostas são pontuadas de 1 a 4, sendo as maiores pontuações indicativas de uso problemático da substância. Classifica-se o usuário em uma de quatro zonas de risco de acordo com o escore obtido: zona I (até 7 pontos: indica uso de baixo risco ou abstinência); zona II (de 8 a 15 pontos: indica uso de risco); zona III (de 16 a 19 pontos: sugere uso nocivo) e zona IV (acima de 20 pontos: mostra uma possível dependência). Foi validado em diversos países, inclusive no Brasil, apresentando bons níveis de sensibilidade $(87,8 \%)$ e especificidade (81\%) para detecção do uso abusivo desta substância. ${ }^{29,30}$

Foi utilizado o questionário sobre histórico de quedas, contendo as perguntas: 77- "No último ano o(a) Sr/Sra sofreu alguma queda? Se sim, quantas?" e, por fim, o questionário sobre o uso de Serviços de Saúde, contendo as perguntas: 99- O(A) senhor(a) precisou ser internado(a) no hospital pelo menos por uma noite?

\section{Análise estatística dos dados}

A análise das informações coletadas por meio dos formulários de entrevistas da rede FIBRA-JF foi feita inicialmente no programa estatístico Statistical Package for the Social Sciences (SPSS) versão 20.0. Para a análise posterior foi construído um banco de dados pela tabulação e codificação dos dados que foram analisados pelo programa Sphinx lexica \& eureca $v$. 5, estabelecendo a frequência de cada variável na amostra levantada.

Primeiramente foi feita uma análise descritiva das variáveis de interesse anteriormente citadas analisando média, desvio padrão e mediana. Em seguida foi feita uma correlação utilizando o teste de Pearson por meio da análise de dois grupos (homens e mulheres), onde se verificou a presença ou ausência do consumo de risco de álcool e sua associação com quedas e ocorrência de internações nos idosos, ou seja, como a amostra do estudo reagiu ao consumo de álcool em relação a estes dois desfechos.

\section{Resultados}

A amostra foi composta por 128 idosos que relataram consumir álcool, sendo que destes 73 eram do gênero feminino e 55 do gênero masculino.

Os resultados obtidos demonstraram que não houve diferença significativa no consumo de risco de bebidas alcoólicas tanto para homens $(\mathrm{n}=16,51 \%)$ quanto para mulheres $(n=37,16 \%)$ para $(p<0,05)$ (Tabelas 1 e 2$)$.

Observou-se que não houve correlação entre consumo de risco e quedas no grupo masculino ( $\mathrm{p}=69,41 \%)$ (Tabela 3).

No entanto, observou-se uma diferença estatisticamente significativa entre o número de quedas e o consumo de risco de álcool no grupo feminino ( $\mathrm{p}=99,55 \%)$. Os idosos que consumiram de 2 a 3 doses por dia sofreram 3 quedas nos últimos 12 meses, e os que consumiram de 4 a 5 doses sofreram 2 quedas nos últimos 12 meses (Tabela 4).

O consumo de risco de álcool em homens e mulheres associado à internação por pelo menos uma noite não apresentou relevância estatisticamente significativa (Tabelas 5 e 6).

\section{Discussão}

O alcoolismo representa um dos maiores problemas de saúde pública. A dependência e o uso nocivo do álcool estão presentes em 6\% dos atendimentos na rede de atenção primária. Na sociedade atual o uso do álcool possui uma conotação diferenciada das demais drogas. Seu caráter lícito, de baixo custo e acesso facilitado lhe fornecem aceitação social, dificultando o enfrentamento acerca de seu uso abusivo. O consumo é francamente estimulado pela indústria do álcool ocupando o primeiro lugar mundial em consumo em relação às substâncias psicoativas, o que dificulta a compreensão como um problema de saúde pública. ${ }^{2,3,8}$

A mortalidade e as deficiências causadas pelo consumo de bebidas alcoólicas superam aquelas ocasionadas pelo tabaco; determinando $3,2 \%$ da mortalidade global, sendo 5,6\% deste valor para homens e 0,6\% em mulheres. ${ }^{10,14,22}$

O uso, abuso e a dependência de substâncias psicoativas entre os idosos têm forte impacto econômico sobre os sistemas de saúde dos países desenvolvidos, gerando carga crescente. Essa tendência tem sido observada também nos países emergentes. Neste contexto, é conhecido que o uso do álcool pode causar prejuízos à saúde do idoso independentemente da frequência ou quantidade ingerida. ${ }^{31}$ Portanto, mesmo nos casos 
Tabela 1. Relação entre consumo de risco de álcool e quedas no gênero masculino

\begin{tabular}{lccc}
\hline Sofreu queda? & \multicolumn{1}{c}{$\operatorname{Sim}$} & Não & Total \\
\hline Quantas doses num dia normal? & & & $100 \%(32)$ \\
\hline ou 1 & $31,3 \%(10)$ & $68,75 \%(22)$ & $100 \%(20)$ \\
\hline 2 ou 3 & $25,0 \%(5)$ & $75 \%(15)$ & $100 \%(52)$ \\
\hline Total & $27,3 \%(15)$ & $71,15 \%(37)$ & 100 \\
\hline
\end{tabular}

Produzido pelos autores. A dependência não é significativa. Qui $=0,23, g l=1,1-p=37,16 \%$. Odds-ratio: 1,36

Tabela 2. Relação entre consumo de risco de álcool e quedas no gênero feminino

\begin{tabular}{lccc}
\hline Sofreu queda? & Sim & Não & Total \\
\hline Quantas doses num dia normal? & & & $100 \%(63)$ \\
0 ou 1 & $33,3 \%(21)$ & $66,67 \%(42)$ & $100 \%(10)$ \\
\hline 2 ou 3 & $30,0 \%(3)$ & $70 \%(7)$ & $100 \%(73)$ \\
\hline Total & $32,9 \%(24)$ & $67,12 \%(49)$ & \\
\hline
\end{tabular}

Produzido pelos autores. A dependência não é significativa. Quii ${ }^{2}=0,04, g l=1,1-p=16,51 \%$. Odd-ratio: 1,17

Tabela 3. Relação entre o número de doses de álcool e o número de quedas no gênero masculino

\begin{tabular}{lccccc}
\hline Número de quedas? & 0 & 1 & 2 & 4 & Total \\
\hline Quantas doses num dia normal? & & & & & \\
\hline ou 1 & $68,8 \%(22)$ & $21,87 \%(7)$ & $9,37 \%(3)$ & $0 \%(0)$ & $100 \%(32)$ \\
\hline 2 ou 3 & $78,57 \%(11)$ & $7,14 \%(1)$ & $7,14 \%(1)$ & $7,14 \%(1)$ & $100 \%(14)$ \\
\hline 4 ou 5 & $66,7 \%(4)$ & $33,3 \%(2)$ & $0 \%(0)$ & $0 \%(0)$ & $100 \%(6)$ \\
\hline 6 ou 7 & $100 \%(1)$ & $0 \%(0)$ & $0 \%(0)$ & $0 \%(0)$ & $100 \%(1)$ \\
\hline ou mais & $100 \%(2)$ & $0 \%(0)$ & $0 \%(0)$ & $0 \%(0)$ & $100 \%(2)$ \\
\hline Total & $72,7 \%(40)$ & $18,18 \%(10)$ & $7,27 \%(4)$ & $1,82 \%(1)$ & $100 \%(55)$ \\
\hline
\end{tabular}

Produzido pelos autores. A dependência não é significativa. Qui ${ }^{2}=17,22, g \mid=15,1-p=69,41 \%$.

Tabela 4. Relação entre o número de doses de álcool e número de quedas no gênero feminino

\begin{tabular}{lcccccc}
\hline Número de quedas? & 0 & 1 & 2 & 4 & 5 & Total \\
\hline Quantas doses num dia normal? & & & & & & \\
\hline ou 1 & $65,1 \%(41)$ & $22,22 \%(14)$ & $9,52 \%(6)$ & $0 \%(0)$ & $3,17 \%(2)$ & $100 \%(63)$ \\
\hline ou 3 & $85,7 \%(6)$ & $0 \%(0)$ & $0 \%(0)$ & $14,28 \%(1)$ & $0 \%(0)$ & $100 \%(7)$ \\
\hline ou 5 & $33,3 \%(1)$ & $0 \%(0)$ & $66,7 \%(2)$ & $0 \%(0)$ & $0 \%(0)$ & $100 \%(3)$ \\
\hline Total & $65,8 \%(48)$ & $19,17 \%(14)$ & $10,95 \%(8)$ & $1,36 \%(1)$ & $2,73 \%(2)$ & $100 \%(73)$ \\
\hline
\end{tabular}

Produzido pelos autores. A dependência é muito significativa. $Q \mathrm{ui}^{2}=22,26, \mathrm{gl}=8,1-\mathrm{p}=99,55 \%$. 


\section{Artigo original}

Tabela 5. Relação entre consumo de risco de álcool e internações no gênero masculino

\begin{tabular}{lccc}
\hline Foi internado? & Sim & Não & Total \\
\hline Quantas doses num dia normal? & & & $100 \%$ \\
\hline ou 1 & $12,5 \%$ & $87,5 \%$ & $100 \%$ \\
\hline 2 ou 3 & $8,7 \%$ & $91,3 \%$ & $100 \%$ \\
\hline Total & $10,9 \%$ & $89,1 \%$ & \\
\hline
\end{tabular}

Produzido pelos autores. A dependência não é significativa. $Q u^{2}{ }^{2}=0,20, g l=1,1-p=34,47 \%$.

Tabela 6. Relação entre consumo de risco de álcool e internações no gênero feminino

\begin{tabular}{lccc}
\hline Foi internado? & Sim & Não & Total \\
\hline Quantas doses num dia normal? & & & $100 \%$ \\
\hline ou 1 & $15,9 \%$ & $84,1 \%$ & $100 \%$ \\
\hline 2 ou 3 & $20 \%$ & $80 \%$ & $100 \%$ \\
\hline Total & $16,4 \%$ & $83,6 \%$ & \\
\hline
\end{tabular}

Produzido pelos autores. A dependência não é significativa. $Q \mathrm{ui}^{2}=0,11, \mathrm{gl}=1,1-\mathrm{p}=25,64 \%$.

em que o idoso consome bebida alcoólica apenas esporadicamente, pode existir uma relação direta entre o uso e os danos para a saúde, o que pode caracterizar o consumo de álcool na terceira idade como um comportamento de risco. ${ }^{32}$

Sua ação prolongada sobre o sistema nervoso central faz com que alcoólicos crônicos apresentem muitas vezes dificuldades em testes simples de equilíbrio e também quando caminham, manifestando uma marcha atáxica com base alargada. Um leve esforço exigido para alcoólicos crônicos com lesão cerebelar muito provavelmente levará a uma perda súbita de equilíbrio, mostrando sua falta de mecanismos adaptativos para correção deste deficit.15,19

O consumo abusivo do álcool traz consequências para a mulher em vários aspectos, podendo interferir nos níveis estrogênicos e sendo associado ao aparecimento da osteoporose, tendo em vista que mulheres alcoolistas apresentam frequentemente hipocalcemia, hipomagnesemia e hipoparatireoidismo, acarretando em disfunções que levam à osteoporose. ${ }^{33,34}$ Esses fatores vêm contribuir para o evento queda nas mulheres, de acordo com os resultados deste estudo, onde mulheres que apresentavam um padrão de consumo de risco de álcool sofreram mais quedas. Já no grupo masculino, o qual também se adequou ao consumo de risco, não apresentou relevância sig-nificativa em relação às quedas.
Conforme aponta a literatura, as mulheres usuárias de álcool atribuem maior significado do uso a eventos internos, diferente dos homens, que atribuem a eventos relacionados ao trabalho. Além disso, elas são potencialmente mais sensíveis aos assuntos relativos às questões domésticas e de suas vidas íntimas. ${ }^{35}$

Os transtornos relacionados ao uso do álcool representam ainda a oitava causa de concessão de auxílio-doença no sistema previdenciário brasileiro, sendo que os pacientes com problemas relacionados a essa substância utilizam os serviços de saúde até três vezes mais. ${ }^{36}$

Neste estudo a relação entre as variáveis consumo de risco de álcool e internação não foi significativa, embora seja conhecido que o consumo excessivo de álcool está associado ao aumento da mortalidade e de agravos adversos à saúde. Torna-se razoável, portanto, supor uma associação causal entre o consumo de álcool e o aumento da utilização e do custo da assistência em saúde, entre os quais a internação. ${ }^{37,38}$

Estudos demonstram que o tempo de internação e o custo do tratamento hospitalar têm uma relação diretamente proporcional com o consumo de álcool. Ambos foram mais elevados em indivíduos que consumiram mais de $450 \mathrm{~g} / \mathrm{semana}$ e menores para os indivíduos que consomem $150-299 \mathrm{~g} / \mathrm{semana}^{38}$

A internação ou hospitalização é considerada de grande risco especialmente para as pessoas mais 
idosas. Os efeitos da restrição ao leito na musculatura e na massa óssea do idoso durante a internação são devastadores e podem levar à perda da capacidade de deambulação, além de aumentar o risco de fraturas. ${ }^{21-23,33,34}$ Além disso, medicações psicotrópicas podem reduzir a percepção da necessidade de urinar, vindo o paciente a desenvolver quadros de incontinência urinária. ${ }^{38}$ Durante a internação, os pacientes idosos podem sofrer privação sensorial causada por deficit visual por presbiopia, catarata, glaucoma, deficit auditivo ou mesmo falta de óculos e próteses auditivas, comumente esquecidas em casa ou indevidamente proibidas em alguns ambientes hospitalares como unidades de terapia intensiva. ${ }^{39}$ Tais alterações causam importante deficit de equilíbrio levando às quedas. ${ }^{40}$

Os resultados do estudo de Lima et al auxiliam nesta discussão pelo fato de não se poder inferir causalidade na associação entre o consumo de álcool, quedas e idade avançada. No entanto, tal associação embasa a hipótese de que o uso abusivo de álcool encontra-se relacionado a agravos na saúde desta população. Nesta direção, os resultados do presente estudo indicam que o consumo de risco de bebidas alcoólicas em idosos pode estar relacionado com maior ocorrência de quedas nesta população, principalmente nas mulheres devido a diversos fatores biológicos e metabólicos tais como a osteoporose. ${ }^{33,} 34$ Sendo assim, as mulheres possuem maior predisposição a sofrer quedas. ${ }^{12}$

O estudo de Barbosa et al indica associação entre o consumo de álcool no sexo masculino e fragilidade. ${ }^{22}$ Outro aspecto é que a população analisada viveu em um período em que não era aceito culturalmente pela sociedade que mulheres fossem consumidoras habituais de álcool. O fato de não haver relevância significativa na relação de consumo de álcool e internações pode ser justificado pela predominância feminina no banco de dados utilizado. Além disso, é consenso que as mulheres têm uma maior preocupação com a saúde em relação aos homens, o que poderia explicar um efeito dispersivo na associação dos dados em relação ao consumo de risco e internação.

As limitações identificadas no presente estudo e que podem ter influenciado os resultados do trabalho foram três. A característica de delineamento transversal dos dados não permite avaliar a história de vida prévia da população. Por isso não foi possível identificar se os idosos apresentavam um histórico de alcoolismo crônico de longa data, reduzindo o poder de acurácia nas relações estudadas. Em segundo lugar, a população analisada viveu grande parte de suas vidas em um período em que o consumo de álcool não era tão influenciado pela indústria, ou seja, um consumo considerado inferior ao observado nos dias atuais. Por fim, como já citado, a predominância do sexo feminino no banco de dados pode ter influenciado os resultados das análises.

Novos estudos longitudinais devem ser encorajados para avaliar de forma mais precisa a relação entre alcoolismo e seus desfechos adversos na população idosa.

\section{Referências}

1. Balbinot $A D$, Horta RL, Costa JSD, et al. Hospitalizações por uso de drogas não se alteram com uma década de Reforma Psiquiátrica. Rev Saude Publica. 2016;50:2.

2. Organização Mundial da Saúde (OMS). OMS, 2017 Estatísticas mundiais de saúde 2017: Monitoramento da saúde para os Objetivos de Desenvolvimento Sustentável (ODS). Genebra:OMS; 2017.

3. Organização Mundial da Saúde (OMS). CISA - Centro de Informações sobre Saúde e Álcool. Relatório Global sobre Álcool e Saúde-2014. Genebra:OMS; 2014.

4. Ceylan-Isik AF, McBride SM, Ren J. Sex diference in alcoholism: who is at a greater risk for development o alcoholic complication? Life Sciences. [S.I.]. 2010;86:136-138.

5. Scheffer M, Almeida RMM. Consumo de álcool e diferenças entre homens e mulheres: comportamento impulsivo, aspectos cognitivos e neuroquímicos. Revista Neuropsicologia Latinoamericana. 2010;2(3):1-11.

6. Oliveira JB, Kerr-Corrêa F, Lima MCP, et al. Validity of alcohol screening instruments in general population gender studies: an analytical review. Current drug abuse reviews. 2014;7(1):59-65.

7. Wolle CC, Sanches M, Zilberman ML et al. Differences in drinking patterns between men and women in Brazil. Rev. Bras. Psiquiatr. [Internet]. 2011 Dec [cited 2018 May 07];33(4):367-373.

8. Wilsnack R, Wilsnack S, Obot I. Why study gender, alcohol and culture. Alcohol, gender and drinking problems: perspectives from low and middle income countries.[Geneva]. World Health Organization. 2005;1-23.

9. Oliveira JB, Santos JLF, Kerr-Corrêa F, et al. Alcohol screening instruments in elderly male: a population-based survey in metropolitan São Paulo, Braz Rev Bras Psiquiatr. 2011;33(4):347-352.

10. Grinfeld $H$. Álcool e suas consequências: uma abordagem multiconceitual. Consumo abusivo de álcool durante a gravidez. São Paulo: Editora Manole, 2009;179-99.

11. Immonen S, Valvanne J, Pitkala KH. Alcohol use of older adults: drinking alcohol for medicinal purposes. Age ageing. 2011;40(5):633-6377.

12. Lima MCP, Simão MO, Oliveira JB, et al. Alcohol use and falls among the elderly in Metropolitan São Paulo, Brazil. Cad. Saúde Pública [Internet]. 2009 Dec [cited 2018 May 07];25(12):26032611.

13. Jonas LT, Silva JV, Mendes MA. Construção da escala avaliativa do risco de quedas para pessoas idosas não institucionalizadas Rev enferm UFPE on line., 2015 Recife, 9(Supl. 4):7977-85.

14. Halme, JT, Seppä, K, Alho, H, et al. Alcohol consumption and allcause mortality among elderly in Finland. Drug Alcohol Depend. 2010;106(2-3):212-218.

15. Lopes MA, Furtado EF, Ferrioli E, et al. Prevalence of alcohol-related problems in an elderly population and their association 


\section{Artigo original}

with cognitive impairment and dementia. Alcohol Clin Exp Res. 2010;34(4):726-733.

16. Peters R, Peters J, Warner J, et al. Alcohol, dementia and cognitive decline in the elderly: a systematic review. Age Ageing. 2008;37:505-512.

17. Lane SD, da Costa SC, Teixeira AL, et al. The impact of substance use disorders on clinical outcomes in older-adult psychiatric inpatients. Int J Geriatr Psychiatry. 2018 Feb;33(2):e323-329.

18. Souza JG, Jones KM, Fonseca AD, et al. Consumption profile and factors associated with the ingestion of beer and distilled beverages among elderly Brazilians: Gender differences. Geriatr Gerontol Int. 2016;16(7):810-20. doi: 10.1111/ggi.12556. Epub 2015 Aug 5.

19. Cruz DT, Ribeiro LC, Vieira MT, et al. Prevalência de quedas e fatores associados em idosos. Rev Saúde Pública. 2012; 46(1):138-46.

20. Falsarella CR, Gasparotto LPR, Coimbra AMV. Quedas: conceitos, frequências e aplicações à assistência ao idoso. Revisão da literatura. Rev Bras Geriatr Gerontol. 2014;17(4):897-910.

21. Pillon SC, Santos MA, Kano MY, et al. Registros de óbitos e internações por transtornos relacionados ao uso de álcool em idosos Rev. enferm. UERJ, Rio de Janeiro, 2011;19(4):536-40.

22. Barbosa MB, Pereira CV, Cruz DT, et al. Prevalência e fatores associados ao consumo de álcool e de tabaco em idosos não institucionalizados. Rev Bras Geriatr Gerontol. Rio de Janeiro, 2018; 21(2):125-135.

23. Chamberlain AM, Sauver JL, Jacobson DJ, et al. Social and behavioural factors associated with frailty trajectories in a population-based cohort of older adults. BMJ Open. 2016;6:1-11.

24. Remor CB, Bós AJG, Werlang MC. Características relacionadas ao perfil de fragilidade no idoso. Sci Med. 2011; 21(3):107-112.

25. Roerecke M, Rehm J. Cause-specific mortality risk in alcohol use disorder treatment patients: a systematic review and meta-analysis. Int. J. Epidemiol. 2014;43(3):906-919.

26. Lourenço RA, Moreira VLG, Banhato EFC, et al. Prevalence of frailty and associated factors in a community- dwelling older people cohort living in Juiz de Fora, Minas Gerais, Brazil: Fibra-JF Study. Ciênc Saúde Coletiva. 2019;24(1):35-44.

27. Santos WS, Gouveia WV, Fernandes DP, et al. Alcohol Use Disorder Identification Test (AUDIT): explorando seus parâmetros psicométricos. J Bras Psiquiatr. [Internet]. 2012 [cited 2018 May 07];61(3):117-123.

28. Meneses-Gaya C, Zuardi AW, Loureiro SR, et al. Alcohol Use Disorders Identification Test (AUDIT): an updated systematic review of psychometric properties. Psychol Neurosci. 2009;2(1):83-97.

29. Babor TF, Higgins-Biddle J, Saunders J, et al. The alcohol use disorders identification test: guidelines for use in primary care. [internet] 2. ed. Genebra: World Health Organization; 2001.

30. Soares SM, Lima EDRP, Naegle MA, et al. Consumo de álcool e qualidade de vida em idosos na saúde da família. R. Enferm. Cent. O. Min. 2016 set/dez; 6(3):2362-2376

31. O'Connell H, Chin AV, Cunningham C, et al. Alcohol use disorders in elderly people: redefining an age old problem in old age. BMJ. 2013; 327(7416):664-7.

32. Schmidt MI, Duncan BB, Azevedo e Silva G, et al. Chronic non-communicable diseases in Brazil: burden and current challenges. Lancet. 2011;377(9781):1949-61. doi: 10.1016/S01406736(11)60135-9. Epub 2011 May 9.

33. Martins GSB. Influência do Tabagismo e Alcoolismo na Densidade Mineral Óssea. Rev Med Saúde Brasília. 2012;1(1):4-9.

34. Moraes LFS, Silva EN, Silva DAS, et al. Gastos com o tratamento da osteoporose em idosos do Brasil (2008 2010): análise dos fatores associados. Rev Bras Epidemiol. 2014;17(3):719-34.

35. Wilsnack R, Wilsnack S. Gender and alcohol individual and social perspectives. Nova Jérsei: Rutgers Center of Alcohol Studies; 1997.

36. Hunkeler EM, Hung YY, Rice DP, et al. Alcohol consumption patterns and health care costs in an HMO. Drug Alcohol Depend. 2001;64(2):181-90.

37. Rao R, Schofield P, Ashworth M. Alcohol use, socioeconomic deprivation and ethnicity in older people. BMJ Open. 2015;5:1-6.

38. Sales MVC, Silva TJA, Gil Jr LA, et al. Efeitos adversos da internação hospitalar para o idoso. Rev Bras Geriatr Gerontol. [S.I.]. 2010;4(4):238-246.

39. Nassisi D, Korc B, Hahn S, et al. The Evaluation and management of the acutely agitated elderly patient. Mt Sinai J Med. 2006 [S.I.];73(7):976-984.

40. Schmidt PMS, Giordani AM, Rossi AG, et al. Avaliação do equilíbrio em alcoólicos. Braz J Otorhinolaryngol. 2010 [S.I.];76:148155. 